\title{
Clozapine is strongly associated with the risk of pneumonia and inflammation
}

\author{
Jose de Leon (i), ${ }^{1}$ Can-Jun Ruan, ${ }^{2}$ Hélène Verdoux, ${ }^{3}$ \\ Chuanyue Wang ${ }^{2}$
}

\section{ABSTRACT}

Clinicians need to remember that (1) systemic inflammations can increase clozapine level; (2) clozapine, by itself, can cause inflammation, particularly during titration that is too rapid for that patient; (3) clozapine may increase the risk of infection; and (4) more specifically, clozapine may be particularly strongly associated with the risk of pneumonia. Pneumonia appears to be associated with high mortality in clozapine patients around the world. Clinicians who are alert to the risk of pneumonia in clozapine patients may significantly decrease mortality in clozapine patients. There is no data on COVID-19 infections in clozapine patients, but based on what we know about clozapine pharmacology, we can hypothesise that clozapine, possibly by impairing immunological mechanisms, may increase the risk of pneumonia in infected patients. More importantly, once fever and/or pneumonia develops, the clozapine dose should be cut in half to decrease the risk of clozapine intoxication. If there is any doubt that in spite of halving the dose there are still signs of clozapine intoxication, completely stopping clozapine may be indicated. Once the signs of inflammation and fever have disappeared, the clozapine dose can be increased to the prior dosage level.

\section{INTRODUCTION}

This forum article reviews the multifaceted relationship of clozapine and inflammation and its impact on the clinical care of patients. After briefly reviewing clozapine metabolism, four major topics are discussed: (1) inflammation can increase clozapine levels; (2) clozapine can cause inflammation, particularly during titration; (3) clozapine may increase the risk of infection; and (4) more specifically,

\footnotetext{
${ }^{1}$ University of Kentucky Mental Health Research Center, Eastern State Hospital, Lexington, Kentucky, USA

${ }^{2}$ Beijing Key Laboratory of Mental Disorders, Beijing Anding Hospital, Beijing, China

${ }^{3}$ Inserm, Bordeaux Population Health Research Center, University of Bordeaux Faculty of Medical Sciences, Bordeaux, Aquitaine, France

Correspondence to Dr Jose de Leon; jdeleon@uky.edu
}

clozapine may be particularly strongly associated with the risk of pneumonia.

This data provides a consistent pattern, but the studies are limited by their observational nature. Obviously for ethical reasons, it is not possible to randomise clozapine patients to infections versus placebo.

There is no data on COVID-19 in clozapine patients, but based on what we know about clozapine pharmacology, we can hypothesise that clozapine, possibly by impairing immunological mechanisms, may increase the risk of pneumonia in infected patients. More importantly, once fever and/or pneumonia develops, the clozapine dose should be cut in half to decrease the risk of clozapine intoxication. If there is any doubt that in spite of halving the dose there are still signs of clozapine intoxication, completely stopping clozapine may be indicated. Once the signs of inflammation and fever have disappeared, the clozapine dose can be increased to the prior dosage level.

\section{CLOZAPINE METABOLISM}

In 1989 before pharmacokinetic studies were required, the United States (US) Food and Drug Administration (FDA) approved clozapine with very limited information on clozapine metabolism. In 1994, Bertilsson et $a l^{1}$ described CYP1A2 as its major metabolic pathway. CYP1A2 pharmacology is highly relevant in clozapine dosing. Norclozapine is the main metabolite of clozapine and appears to be mainly eliminated by the kidney, since its conjugated metabolites are present in the urine and the serum free norclozapine is excreted by an unknown renal transporter that can be inhibited by gemfibrozil. With geriatric age, clozapine clearance from the body decreases; this is probably explained by the decrease in renal function and subsequent decrease in renal clearance of norclozapine and other metabolites eliminated in the urine.

Tobacco smoke has polycyclic aromatic hydrocarbons which bind to the aryl hydrocarbon receptor and induce CYP1A2 expression, increasing the levels of CYP1A2, which is mainly expressed in the liver. Therefore, smokers tend to have serum concentration values that are approximately 0.80 that of non-smokers when using the same clozapine dose. ${ }^{2}$ Conversely, oestrogens have inhibitory effects on CYP1A2 activity. Male patients, then, tend to have serum concentration values that are approximately 0.86 that of females when using the same clozapine dose. ${ }^{2}$ Co-medication with inducers such as carbamazepine, phenytoin or rifampicin increase clozapine metabolism and decrease clozapine levels. The most important inhibitors of clozapine metabolism are ciprofloxacin, oral contraceptives, fluvoxamine and caffeine in high doses. Valproic acid can be an inducer and/or an inhibitor of clozapine metabolism; this varies from patient to patient and over time. During clozapine titration, clinicians should be more concerned about the potential of valproic acid to act as an inhibitor.

Clozapine is prone to cause side effects, called adverse drug reactions (ADRs) by pharmacologists. Some of these clozapine ADRs, such as sedation, hypersalivation, constipation and seizures, are dose-related. It is probably more accurate to describe them as serum concentration-related. An expert guideline recommends for efficacy in schizophrenia trough steady-state clozapine concentrations of $350-600 \mathrm{ng} / \mathrm{mL} .{ }^{3}$ This indicates a narrow therapeutic index. The serum concentration of norclozapine does not contribute to antipsychotic efficacy since norclozapine has no antipsychotic activity. On the other hand, it is possible that serum norclozapine concentration may contribute to most or all clozapine ADRs.

If clinicians have access to clozapine levels, they should use them to maintain each clozapine patient on the 
lowest dose possible that provides stable trough steady-state clozapine levels within the therapeutic range. Assuming that the patient practices good medical adherence and is reliable, we recommend establishing a clozapine dose that provides stable serum concentrations between 350 and $400 \mathrm{ng} / \mathrm{mL}$, to allow some room for normal fluctuations in the clinical environment. To increase compliance and reduce daily ADRs, a single administration at night can be used.

Clozapine dosing is not only influenced by geriatric age, smoking, gender and co-medication but ethnicity (Asian vs non-Asian). In 1997, Asian researchers first described Chinese patients as having serum concentrations similar to those of Caucasians with only half the clozapine dose used in the US. ${ }^{45}$ A meta-analytic review ${ }^{2}$ showed that Chinese and other East Asians have lower clozapine clearance than Caucasians. The lower clozapine clearance, probably explained by lower CYP1A2 activity, appears to be present not only in Chinese patients but is also common to other Asians. ${ }^{6}$ The FDA describes Asians as those people whose ancestral origins range geographically from Pakistan to Japan. ${ }^{6}$ In Asians, to reach $350 \mathrm{ng} / \mathrm{mL}$, a non-smoking female with average metabolism needs around $150 \mathrm{mg} /$ day and a male smoker $300 \mathrm{mg} /$ day, requiring typical doses in Asians from 150 to $300 \mathrm{mg} /$ day. $^{6}$ On the other hand, a non-smoking US Caucasian female with average clozapine metabolism needs around $300 \mathrm{mg} /$ day while a US Caucasian male smoker needs $600 \mathrm{mg} /$ day, requiring typical doses in the US from 300 to $600 \mathrm{mg} /$ day. $^{6}$

To define clozapine poor metabolisers (PMs), it is necessary to stratify Asians versus non-Asians. Within the ethnic group, taking powerful clozapine inhibitors and/or being severely obese can be explained as being a phenotypic PM. In five Asian samples, around $7 \%$ of the patients (2\%-13\%) were phenotypic PMs, with no known cause, but four of the five studies were limited by not ruling out current inflammation, which can make a patient look temporarily like a clozapine PM. ${ }^{7}$ Thus, it is possible that around $7 \%(2 \%-13 \%)$ of Asians may have unknown genetic mutations possibly at the CYP1A2 gene associated with being a clozapine PM. ${ }^{7}$

Until there is a better definition of clozapine PMs, we recommend considering Asian clozapine PMs as those who reach concentrations of $350 \mathrm{ng} / \mathrm{mL}$ with clozapine doses $<150 \mathrm{mg} /$ day. A more precise definition, including smoking and gender stratification, is provided in a review article. ${ }^{6}$ Asian female non-smokers who are PMs need a clozapine dose around $50 \mathrm{mg} /$ day to reach $350 \mathrm{ng} /$ $\mathrm{mL}$, while Asian male smokers who are PMs need a clozapine dose around $125 \mathrm{mg}$ /day. ${ }^{6}$ Until a better definition of clozapine PMs in nonAsians is established, we recommend considering non-Asian clozapine PMs as those who reach $350 \mathrm{ng} / \mathrm{mL}$ with clozapine doses $<300 \mathrm{mg} /$ day. $^{2}$

\section{INFLAMMATION CAN INCREASE CLOZAPINE LEVELS}

The US package insert for theophylline, a drug metabolised by CYP1A2, indicates that upper respiratory infections with fever increase serum theophylline concentrations. The release of cytokines during the infection decreases the activity and/or expression of CYP1A2. More relevant for clinicians, experts on theophylline ${ }^{8}$ recommended that the theophylline dose should be approximately halved to avoid intoxication during these infections. Therefore, the first author reduced the clozapine dose by half ${ }^{8}$ when he first diagnosed a clozapine intoxication during an upper respiratory infection with fever in one of his patients. The later arrival of the clozapine serum concentration level verified his diagnosis of clozapine intoxication. ${ }^{8}$ In 2004, based on this case $^{9}$ and the available literature, he proposed that during severe inflammations/infections, including pneumonia, the clozapine dose should be halved until clozapine levels are available to better personalise clozapine dosing. ${ }^{9}$ Through 2016, there are 40 published cases of clozapine elevation during infections. ${ }^{10}$ There are also published clozapine intoxications during severe inflammation without infection. ${ }^{11}$

More recently, in a Beijing hospital, three cases of clozapine intoxication we identified included ones with pneumonia, ${ }^{12}$ influenza ${ }^{11}$ and dermatitis in the absence of infection. ${ }^{11}$ In these three Chinese patients severe infections were associated with roughly double the serum concentrations, ${ }^{1112}$ while the effects in relation to dermatitis depended more on its severity. A doubling of the concentration was only observed when there was an elevation of the serum C-reactive protein (CRP) and widespread effect on the skin all over the body. ${ }^{11}$ In a recent retrospective review of 131 clozapine inpatients at Beijing Anding Hospital, we found 18 episodes of infections/ inflammations in 16 patients. At the total sample level, these episodes: (1) extended for $2 \%(482 / 24789)$ of clozapine days and (2) contaminated $3 \%(46 / 1384)$ of trough steady-state serum concentrations of clozapine. At the individual level, we found: (1) no clinically relevant effects on the serum clozapine concentrations in the $11 \%$ of infection episodes which presented with no leukocytosis or CRP elevations; (2) halving the clozapine dose would be advisable in $61 \%$ of the infection episodes; and (3) reducing the clozapine dose to one-third would be advisable in $28 \%$ of infection episodes. ${ }^{13}$

Therefore, based on the $\mathrm{US}^{8}{ }^{14}$ and Chinese experiences ${ }^{11-13}$ as well as on the literature, ${ }^{10}$ we proposed that clinicians need to be very alert regarding clozapine patients' risk of clozapine intoxications during any kind of systemic inflammation associated with fever and/or CRP elevations. Until better data is available, based on our experience in the US and China, we have developed three sets of recommendations regarding infection, based on timeframe: (1) prevention; (2) during the infection; and (3) after the infection. ${ }^{13}$

For prevention, we recommend that psychiatrists using clozapine should educate their outpatients and families to be attentive to signs or symptoms of infection/inflammation 
or fever and to contact them immediately to prevent clozapine intoxications. Moreover, a careful clinician, after reaching a stable maintenance clozapine dose, should go ahead and measure two or three trough and steady-state serum clozapine concentrations. By calculating the mean concentration and correcting by the clozapine dose ${ }^{2}$ the clinician can establish a baseline for clozapine metabolism in that patient and the lowest clozapine dose providing a therapeutic serum concentration. In the unfortunate event that the patient develops a systemic infection or inflammation, this baseline clozapine metabolism can be compared with the decreased metabolism during the inflammation.

Once an infection has developed, the psychiatrist should order a CRP level. When fever and/or CRP elevations develop, the psychiatrist should consider immediately halving the clozapine dose and monitor for signs of clozapine intoxication. If the clinician has access to clozapine Therapeutic drug monotoring (TDM) when the lab returns the clozapine TDM, it will be possible to better adjust the dosage. If signs of clozapine intoxication are already present it may be safer to stop clozapine for 2-3 days or until the serum clozapine concentration report arrives.

After the infection/inflammation has resolved and the CRP has normalised, we recommend going back to the prior clozapine dose without uptitration, since half of the dose during infection will provide roughly the same concentration as the dose without infection. If the clinician did not measure clozapine concentrations before the infection, it may be wise to measure them after the infection to establish the lowest clozapine dose providing therapeutic serum concentrations and efficacy for maintenance treatment.

Other antipsychotics may be associated with elevations in serum concentrations during infections or inflammations, particularly those metabolised by CYP1A2 and CYP3A4. Olanzapine is mainly metabolised by CYP1A2 and was associated with increases in serum concentrations during an upper respiratory infection in a case report ${ }^{8}$ but it has a wider therapeutic index than clozapine. Some case reports and observational studies previously reviewed in another article $^{11}$ suggest that infections can increase the serum concentrations of antipsychotics metabolised by CYP3A4. Three second-generation antipsychotics are mainly metabolised by CYP3A4: cariprazine, quetiapine and lurasidone. Four second-generation antipsychotics are metabolised by CYP2D 6 and CYP3A4: aripiprazole, brexpiprazole, iloperidone and risperidone. Until better studies are available, we recommend that clinicians remain aware of this potential for increases in serum concentration levels during infections/inflammations and the wisdom of measuring the serum concentrations of these seven antipsychotics metabolised by CYP3A4 when possible.

\section{CLOZAPINE CAN CAUSE INFLAMMATION}

Clozapine can also cause inflammation, although the mechanisms are not completely understood. Most cases of clozapine-induced inflammation occur during titration and the most typical manifestations are CRP elevations, fever and/or myocarditis. ${ }^{15}$ Other rarer forms have been described and include serositis, pneumonitis/alveolitis, hepatitis, pancreatitis, nephritis, colitis and dermatological disorders. ${ }^{15}$

Australia appears to have almost 10-100 times more clozapine-induced myocarditis than European countries. Drug agencies from various countries send their data to a WHO database called VigiBase. ${ }^{16}$ In July 2019, there were 3048 reports of myocarditis associated with clozapine, which resulted in $6 \%$ lethality (170 fatal outcomes). Australia provided $>1500$ of those reports. Most individual countries from continental Europe provided $<30$ reports. In the view of Australian experts, ${ }^{17}$ a $3 \%$ incidence rate of clozapine-induced myocarditis in Australia is explained solely by their intensive cardiac monitoring during titration, which is not practiced in other countries. Psychiatrists in continental Europe, on the other hand, titrate their patients slowly and rarely diagnose clozapine-induced myocarditis. In a real-world study with 3262 clozapine patients from the Danish registry, Rohde $e$ t $a l^{18}$ found a $0.03 \%$ incidence of myocarditis within the first 2 months of treatment. Based on a 3\% incidence of myocarditis according to Australian data and a $6 \%$ death rate due to lack of cardiac monitoring, six deaths $(3 \%$ multiplied by $6 \%$ is $0.18 \%$ and $0.18 \%$ of 3262 is six deaths) were expected in that Danish study, but no myocarditis deaths were identified within the first 2 months of clozapine treatment. ${ }^{18}$

To understand the paradox of clozapine myocarditis, one needs to remember that inflammation decreases clozapine metabolism and then realise that clozapine can also cause inflammation. The history of lamotrigine-induced Stevens-Johnson syndrome provides further understanding. ${ }^{19}$ Lamotrigine-induced Stevens-Johnson syndrome was associated with rapid dose escalation and/ or normal titration in patients taking an inhibitor, such as valproic acid. The first sign that the titration is too fast for that patient is usually a skin rash. If the escalation continues, autoantibodies develop, leading to fullblown Stevens-Johnson syndrome. Therefore, after the first approval, the pharmaceutical company had to reformulate the lamotrigine titration by halving the dose during co-prescription of valproic acid. Similarly, we propose that, first, a clozapine titration that is too fast leads to CRP elevations and/or fever; second, cytokine release decreases clozapine metabolism and this causes a positive feedback mechanism; Third, if the clozapine titration continues, the inflammation evolves into myocarditis, probably with the development of auto-antibodies.

This model was supported by five published cases of clozapine-induced myocarditis in New York. ${ }^{20}$ After their data was provided to the first author, four cases were found to have rapid titration and the other case could 
not tolerate a low clozapine dose of $25 \mathrm{mg}$ /day, which is compatible with being a US clozapine PM. In two of the five patients clozapine levels were available and they were compatible with a decrease in clozapine clearance. $^{20}$

Following this model, clozapineinduced myocarditis can be prevented by using slow, personalised titration: (1) with further slowing for Asians treated by Western psychiatrists, including Australians, who were trained to use Caucasian-level titration in all their patients; (2) considering reversible cases of clozapine PM status by stopping the co-prescription of inhibitors, such as valproic acid and oral contraceptives, and requiring normal CRP before starting clozapine; and (3) considering extremely slow titration for cases of clozapine PMs in which the cause cannot be removed: extreme obesity or an absolutely needed co-prescribed inhibitor. When clozapine dose escalation is associated with an abnormal CRP, cytokines are being released and the titration is too fast for that individual patient. Clozapine dose escalation needs to be held until CRP is normal.

To avoid the risk of myocarditis in Asians, we recommend starting with $12.5 \mathrm{mg}$ as the first dose, followed by targets of $50 \mathrm{mg} /$ day on day 7 , $100 \mathrm{mg} /$ day on day 14 and $150 \mathrm{mg}$ / day on day 21. Moreover, Asian companies producing clozapine should consider developing tables of 5 or $10 \mathrm{mg}$ to facilitate slower titrations for Asian clozapine PMs. ${ }^{6}$ In the absence of formulation with clozapine doses lower than $12.5 \mathrm{mg}$, for Asians with severe obesity or with co-prescription of a medication with potential to be a clinically-relevant inhibitor of clozapine metabolism (eg, valproic acid or an oral contraceptive), we recommend starting with $12.5 \mathrm{mg}$ as the first dose, followed by targets of $25 \mathrm{mg}$ /day on day $7,50 \mathrm{mg}$ / day on day 14 and $75 \mathrm{mg} /$ day on day 21.

In summary, data from (1) the lamotrigine literature; (2) the clinical experience of the authors; (3) the review of TDM studies from the US,
Europe and Asia; and (4) VigiBase has been used to reframe the myocarditis debate. Myocarditis is extremely rare in non-Australian countries and can be avoided by slow, personalised titration. The high incidence of clozapine-induced myocarditis in Australia is probably explained by titration that may be fast for Caucasians and is risky for Asian patients or for Caucasians taking an inhibitor, such as valproic acid.

\section{CLOZAPINE MAY BE ASSOCIATED WITH INCREASED RISK OF INFECTION}

Clinicians all over the world know that on rare occasions clozapine can cause agranulocytosis since this information is included in the clozapine package inserts and most drug agencies require white cell counts to start clozapine. The mechanism is not well understood but it is believed that, in these patients, antibodies are developed against the neutrophils. The peak incidence occurs at 1 month of exposure and declines to negligible levels after 1 year of treatment. ${ }^{21} \mathrm{~A}$ recent meta-analysis provided a prevalence of agranulocytosis of $0.4 \%(95 \%$ CI: $0.3 \%$ to $0.6 \%)$; deaths caused by agranulocytosis were $0.05 \%$ (CI: $0.03 \%$ to $0.09 \%) .{ }^{22}$ This widespread awareness by clinicians is reflected in the almost 35000 reports of neutropenia in VigiBase with the number of fatal outcomes only in the 500s (2\% relative lethality). ${ }^{16}$

More importantly, clinicians may not be aware that even in the absence of neutropenia, clozapine has been associated with a possible increased infection risk. Landry et $a l,{ }^{23}$ based on case reports, proposed that clozapine may increase the risk of tuberculosis and, based on a chart review in a Canadian hospital, may be associated with increased use of antibiotics. Large studies in clozapine patients using the registries of Taiwan ${ }^{24}$ and Denmark ${ }^{25}$ verified an increased risk of tuberculosis with an adjusted risk ratio of 1.63 (95\% CI: 1.10 to 2.40; $\mathrm{p}=0.014)^{24}$ and increased antibiotic use with a relative risk of $1.43(95 \%$ CI: 1.26 to $1.61, \mathrm{p}<0.001) .{ }^{25}$ In a British case-control study, a higher proportion of the clozapine-treated group reported taking more than five courses of antibiotics in the preceding year, $5.3 \%(5 / 123)$ vs $1 \%(1 / 111)$ in controls taking other antipsychotics, and this was associated with an increased percentage of patients with low values for all immunoglobulins. ${ }^{26}$ In an vitro study, clozapine increased the production of the interleukin-1 receptor antagonist. ${ }^{27}$

Regarding possible increased infection risk in the absence of agranulocytosis, we only recommend that clinicians tell clozapine patients to avoid close contact with contagious people.

\section{CLOZAPINE MAY BE MORE STRONGLY ASSOCIATED WITH PNEUMONIA THAN WITH OTHER INFECTIONS}

The second section suggests that infection may be associated with clozapine intoxication and the fourth and prior section that clozapine may increase infection risk possibly by interfering with immunological defenses. As pneumonia is one of the most frequent infections, it is not surprising to find in the literature that pneumonia may be associated with clozapine intoxication and that clozapine, by interfering with immunological mechanisms, may increase the risk of pneumonia. Moreover, attentive reading of the literature suggests that clozapine and pneumonia have strong bidirectional associations beyond what has been described in prior sections.

In 2005, after studying postmarketing surveillance data, the FDA proposed that, by interfering with swallowing, antipsychotics were associated with increases in pneumonia in the elderly and this contributed to mortality in this population. ${ }^{27}$ Although clozapine is rarely used in the elderly, its US package insert, as with other second-generation antipsychotics, was modified to include this warning. Then Kuo et $a l,{ }^{28}$ using the Taiwanese registry, demonstrated that clozapine may be the antipsychotic most closely associated with pneumonia. As a matter of fact, compared with other antipsychotics, clozapine 
may be associated with a greater number of pneumonia cases and greater mortality. ${ }^{16}$ More importantly, clinicians need to be aware that pneumonia may be among the greatest causes of mortality in clozapine patients. VigiBase had $>6000$ reports of broadly-defined pneumonia in clozapine patients with $>2000$ lethal outcomes. To get an accurate perspective, clinicians need to be aware that the broad definitions of agranulocytosis or myocarditis were associated with lethal outcomes in the 500s. ${ }^{16}$

Before describing which known mechanism can explain the strong association between clozapine and pneumonia, we have to acknowledge that in countries other than China clozapine is mainly used for treatment-resistant schizophrenia. Thus, in countries other than China, the association between clozapine and pneumonia may be partly explained by the greater severity of illness in clozapine patients, who are frequently the most treatment-refractory patients with relatively high rates of smoking. Therefore, the contributing effects of the severity level of mental illness on pneumonia needs to be further explored by future studies in clozapine patients. On the other hand, clozapine has specific effects independent of the greater severity of illness in the clozapine patients, since using mirror-image design in the Danish registry, Rhode et $_{\text {al. }}{ }^{29}$

Rhode $e t a l^{29}$ found that clozapine gave the largest absolute increase in pneumonia risk although it did not reach significance, probably due to the relatively small sample size. In the year before clozapine, there were $1.22 \%(23 / 1872)$ patients with pneumonia and in the first year $1.87 \%$ $(35 / 1872)$. This is an increase of $0.64 \%(12 / 1872)(\mathrm{p}=0.10)$.

Clozapine, as with any other antipsychotic, can interfere with swallowing, increasing the potential for aspiration. ${ }^{27}$ The potential for aspiration and aspiration pneumonia during antipsychotic treatment may be further increased by sedation and hypersalivation. ${ }^{27}$ As clozapine is more prone to cause sedation and hypersalivation than other antipsychotics, ${ }^{27}$ it is not surprising that clozapine may be more strongly associated with aspiration pneumonia. Assuming that clozapine is associated with immunological abnormalities, this may also explain an increased risk for other types of pneumonia not associated with aspiration. Rarely, other severe clozapine ADRs, such as severe constipation complicated with ileus, or myocarditis, can be complicated by pneumonia. Once pneumonia develops, clozapine co-prescription may be particularly lethal and worse than other antipsychotics. Severe inflammation during pneumonia releases cytokines that inhibit CYP1A2 expression and/or activity and increase serum clozapine concentrations, further increasing the risk of concentration-related ADRs including hypersalivation, sedation, aspiration or even arrhythmia, creating very dangerous positive feedback. ${ }^{16}$

Our three sets of recommendations for infections in clozapine patients apply to the most important infection, pneumonia. The general preventive measures related to infection apply, including patient/family education and use of the lowest possible dosage providing efficacious response. Specific preventive measures for aspiration pneumonia are close monitoring of sedation and hypersalivation. Clozapine administration should be moved around to the most convenient times in order to increase adherence and decrease these ADRs; treat hypersalivation, preferably with local antimuscarinic treatments which may have less risk of increasing the risk of constipation than oral anticholinergic drugs, such as benztropine, or biperiden. Discontinuation of other co-medications associated with sedation and/or pneumonia risk should be considered. As benzodiazepines are associated with sedation, swallowing disturbances and pneumonia, clinicians may need to consider decreasing or discontinuing them to decrease the aspiration pneumonia risk. Although there is no data, it may not be unreasonable to give pneumonia vaccines to clozapine patients to decrease risk of pneumonia not associated with aspiration, but it cannot be ruled out that vaccines may not be as effective in clozapine patients due to clozapine effects on immunological mechanisms.

Based on our experience with pneumonia, ${ }^{12-14}$ we recommend that clinicians measure trough serum clozapine concentrations to personalise dosing during pneumonia. As the clozapine levels may not be immediately available and due to high lethality, we are convinced that the clozapine dose should be cut in half in all patients with pneumonia. Regarding antibiotic treatment, those such as ciprofloxacin and norfloxacin, which are strong CYP1A2 inhibitors, should not be used.

After the pneumonia has resolved, we make the same recommendation as after infections: return to the prior dose after the CRP has normalised and consider the lowest clozapine dose possible with the fewest ADRs in order to avoid the recurrence of pneumonia in the future.

Clozapine appears to be associated with significantly fewer deaths in patients continuously treated with clozapine compared with other antipsychotics. In a meta-analysis of 24 studies, long-term, crude mortality rate ratios were not significantly lower in patients ever treated with clozapine during follow-up, but significantly lower in patients continuously treated with clozapine compared with patients using other antipsychotics (mortality rate ratio $=0.56,95 \%$ CI: 0.36 to 0.85 , p $=0.007) .{ }^{30}$ By being alert to the multifaceted association of clozapine with inflammation, clinicians may further decrease the mortality in clozapine patients.

In summary, clinicians need to remember that: (1) systemic inflammations can increase clozapine level; (2) clozapine, by itself, can cause inflammation, particularly during titration that is too rapid for that patient; (3) clozapine may increase the risk of infection; and (4) more specifically, clozapine may be particularly strongly associated with the risk of pneumonia. Pneumonia appears to be associated with high mortality in clozapine patients around the world. 
Clinicians who are alert to the risk of pneumonia in clozapine patients may significantly decrease mortality in clozapine patients.

Acknowledgements The authors acknowledge Lorraine Maw, M.A, at the Mental Health Research Center at Eastern State Hospital, Lexington, Kentucky, USA, who helped in editing this article. C-JR is supported by a 2019 NARSAD Young Investigator Award.

Contributors JdL wrote the first draft. All authors (JdL, C-JR, HV and CW) meet criteria for authorship and approved the final manuscript.

Funding The authors have not declared a specific grant for this research from any funding agency in the public, commercial or not-for-profit sectors.

Competing interests None declared.

Patient consent for publication Not required.

Provenance and peer review Commissioned; externally peer reviewed.

\section{(2) OPEN ACCESS}

Open access This is an open access article distributed in accordance with the Creative Commons Attribution Non Commercial (CC BY-NC 4.0) license, which permits others to distribute, remix, adapt, build upon this work non-commercially, and license their derivative works on different terms, provided the original work is properly cited, appropriate credit is given, any changes made indicated, and the use is non-commercial. See: http://creativecommons.org/licenses/by-nc/4.0/.

(c) Author(s) (or their employer(s)) 2020. Re-use permitted under CC BY-NC. No commercial re-use. See rights and permissions. Published by BMJ.

\section{Check for updates}

To cite de Leon J, Ruan C-J, Verdoux H, et al. General Psychiatry Epub ahead of print: [please include Day Month Year]. doi:10.1136/gpsych-2019-100183

Received 9 December 2019

Revised 26 March 2020

Accepted 28 March 2020

General Psychiatry 2020;33:e100183.

doi:10.1136/gpsych-2019-100183

ORCID iD

Jose de Leon http://orcid.org/0000-0002-7756-2314

\section{REFERENCES}

1 Bertilsson L, Carrillo JA, Dahl ML, et al. Clozapine disposition covaries with
CYP1A2 activity determined by a caffeine test. Br J Clin Pharmacol 1994;38:471-3.

2 Ruan C-J, Zang Y-N, Wang C-Y, et al. Clozapine metabolism in East Asians and Caucasians: a pilot exploration of the prevalence of poor metabolizers and a systematic review. J Clin Psychopharmacol 2019;39:135-44.

3 Hiemke C, Bergemann N, Clement $\mathrm{HW}$, et al. Consensus guidelines for therapeutic drug monitoring in Neuropsychopharmacology: update 2017. Pharmacopsychiatry 2018;51:9-62.

4 Chang WH, Lin SK, Lane HY, et al. Clozapine dosages and plasma drug concentrations. J Formos Med Assoc 1997;96:599-605.

5 Chong SA, Tan CH, Khoo YM, et al. Clinical evaluation and plasma clozapine concentrations in Chinese patients with schizophrenia. Ther Drug Monit 1997;19:219-23.

6 de Leon J, Rajkumar AP, Kaithi AR, et al. Do Asian patients require only half of the clozapine dose prescribed for Caucasians? A critical overview. Indian J Psychol Med 2020;42:4-10.

7 Ruan C-J, Wang C-Y, Tang Y-L, et al. Exploring the prevalence of clozapine phenotypic poor metabolizers in 4 Asian samples: they ranged between $2 \%$ and 13 . $J$ Clin Psychopharmacol 2019;39:644-8.

8 de Leon J, Diaz FJ. Serious respiratory infections can increase clozapine levels and contribute to side effects: a case report. Prog Neuropsychopharmacol Biol Psychiatry 2003;27:1059-63.

9 de Leon J. Respiratory infections rather than antibiotics may increase clozapine levels: a critical review of the literature. $J$ Clin Psychiatry 2004;65:1144-5.

10 Clark SR, Warren NS, Kim G, et al. Elevated clozapine levels associated with infection: a systematic review. Schizophr Res 2018;192:50-6.

11 Ruan C-J, Zhang X-L, Guo W, et al. Two cases of high serum clozapine concentrations occurring during inflammation in Chinese patients. Int $J$ Psychiatry Med 2018;53:292-305.

12 Ruan C-J, Zhen X-Y, Ge X-L, et al. Pneumonia can cause clozapine intoxication: a case report. Psychosomatics 2017;58:652-6.

13 Ruan C-J, Zang Y-N, Cheng Y-H, et al. Around $3 \%$ of 1,300 levels were elevated during infections in a retrospective review of 131 beijing hospital in-patients with more than 24,000 days of clozapine treatment. Psychother Psychosom 2020:3

14 Ruan C-J, de Leon J. Thirty years of both ignorance and clinical experience suggest that clozapine intoxication during co-occurring infections and inflammation may have higher morbidity and mortality than is currently believed. Psychosomatics 2019;60:221-2.

15 Verdoux H, Quiles C, de Leon J. Clinical determinants of fever in clozapine users and implications for treatment management: a narrative review. Schizophr Res 2019;211:1-9.

16 De Leon J, Sanz EJ, De Las Cuevas C. Data from the world Health organization's pharmacovigilance database supports the prominent role of pneumonia in mortality associated with clozapine adverse drug reactions. Schizophr Bull 2020:46:1-3.

17 Ronaldson KJ, Fitzgerald PB, McNeil JJ. Clozapine-Induced myocarditis, a widely overlooked adverse reaction. Acta Psychiatr Scand 2015;132:231-40.

18 Rohde C, Polcwiartek C, Kragholm K, et al. Adverse cardiac events in out-patients initiating clozapine treatment: a nationwide register-based study. Acta Psychiatr Scand 2018;137:47-53.

19 Chopra N, de Leon J. Clozapine-Induced myocarditis may be associated with rapid titration: a case report verified with autopsy. Int J Psychiatry Med 2016;51:104-15.

20 de Leon J, Rhee DW, Kondracke A, et al. Rapid titration and decreased clozapine clearance may help explain five cases of clozapine-induced myocarditis in a new York Hospital. Psychosomatics 2020;61:102-3.

21 Myles N, Myles H, Xia S, et al. MetaAnalysis examining the epidemiology of clozapine-associated neutropenia. Acta Psychiatr Scand 2018;138:101-9.

22 Li X-H, Zhong X-M, Lu L, et al. The prevalence of agranulocytosis and related death in clozapine-treated patients: a comprehensive meta-analysis of observational studies. Psychol Med 2020;50:1-12

23 Landry P, Benaliouad F, Tessier S. Increased use of antibiotics in clozapine-treated patients. Int Clin Psychopharmacol 2003;18:297-8.

24 Liu H-C, Hung GC-L, Yang S-Y, et al. Antipsychotic drugs and risk of newly diagnosed tuberculosis in schizophrenia. Psychiatry Clin Neurosci 2018;72:789-800.

25 Nielsen J, Foldager L, Meyer JM. Increased use of antibiotics in patients treated with clozapine. Eur Neuropsychopharmacol 2009;19:483-6.

26 Ponsford M, Castle D, Tahir T, et al. Clozapine is associated with secondary antibody deficiency. Br J Psychiatry 2018. doi:10.1192/bjp.2018.152. [Epub ahead of print: 28 Feb 2020]

27 Cicala G, Barbieri MA, Spina E, et al. A comprehensive review of swallowing difficulties and dysphagia associated with antipsychotics in adults. Expert Rev Clin Pharmacol 2019;12:219-34.

28 Kuo C-J, Yang S-Y, Liao Y-T, et al. SecondGeneration antipsychotic medications and risk of pneumonia in schizophrenia. Schizophr Bull 2013;39:648-57.

29 Rohde C, Siskind D, de Leon J, et al. Antipsychotic medication exposure, clozapine, and pneumonia: results from a self-controlled study. Acta Psychiatr Scand 2019. doi:10.1111/acps.13142. [Epub ahead of print: 25 Dec 2019].

30 Vermeulen JM, van Rooijen G, van de Kerkhof MPJ, et al. Clozapine and long-term mortality risk in patients with schizophrenia: a systematic review and meta-analysis of studies lasting 1.1-12.5 years. Schizophr Bull 2019;45:315-29. 


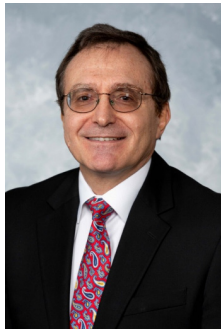

Dr Jose de Leon is a native of Bilbao, Spain. He came to the USA in 1987 to hone his research skills in psychiatry. In 1996 he moved with his family to Lexington, Kentucky, where he became Associate Professor at the University of Kentucky (UK), College of Medicine, Department of Psychiatry. In 2006 he was promoted to Professor. He has served as Medical Director for the Mental Health Research Center at Eastern State Hospital (ESH) since 1996. He developed a set of PowerPoint presentations titled "Training Psychiatrists to Think Like Pharmacologists" to teach the pharmacodynamics and pharmacokinetics of psychiatric drugs using many real psychiatric patient cases. Dr de Leon has mentored research groups around the world. His research career has been prolific. He is an expert in schizophrenia, psychopharmacology, pharmacogenetics and personalized medicine. He has published more than 350 peer-reviewed manuscripts described in PubMed. According to ResearchGate in December of 2019, Dr de Leon's scientific publications have been cited more than 13000 times, and have had more than 40000 reads. He has an h-index of 61 (he has at least 61 articles with $\geq 61$ citations). 